\title{
Is pelvic prophylactic radiotherapy in prostate cancer just right?
}

\author{
Daniel Taussky, Guila Delouya \\ Department of Radiation Oncology, Centre hospitalier de l'Université de Montréal (CHUM), Canada \\ Correspondence to: Daniel Taussky, MD. Department of Radiation Oncology, Centre Hospitalier de l’Université de Montréal - CHUM, 1000, rue St \\ Denis, H2X 0C1, Montreal, Quebec, Canada. Email: daniel.taussky.chum@ssss.gouv.qc.ca. \\ Comment on: Sandler KA, Cook RR, Ciezki JP, et al. Prostate-only versus whole-pelvis radiation with or without a brachytherapy boost for Gleason \\ grade group 5 prostate cancer: a retrospective analysis. Eur Urol 2020;77:3-10.
}

Submitted Apr 21, 2020. Accepted for publication May 11, 2020.

doi: $10.21037 /$ tau-20-881

View this article at: http://dx.doi.org/10.21037/tau-20-881

This present editorial comment accompanies the article by Sandler et al. (1). This article is a valuable contribution to the ongoing discussions on whether and in whom to treat the pelvis of men with prostate cancer getting radiation treatments. A different, less frequent question which seems much more important to us is: why does this discussion continue despite the fact that there are two randomized trials showing no advantage for WPRT but retrospective studies, such as the one by Sandler et al. show a benefit? What could better illustrate the controversy surrounding whole pelvic external beam radiotherapy (WPRT) than the statement made by Avkshto et al. (2) in their very recent publication that "the role of lymph node radiation in modern dose-escalated radiation therapy is a controversial topic". It is noteworthy that their article was not about whether or not to treat the pelvis.

One trial is the Radiation Therapy Oncology Group NRG/ RTOG 9413, last updated in 2018 with a median follow-up of 8.8 years and 14.8 years for surviving patients (3). Patients with localised prostate cancer with an estimated risk of lymph node involvement $>15 \%$ were treated with 4 months of androgen deprivation therapy (ADT) and 70 Gy to the prostate with or without WPRT. The second trial is the French GETUG-01 study, where not all included patients had high risk cancers and therefore not all patients received ADT. Surprisingly, in a post hoc subgroup analysis, only patients with a low risk of lymph node metastasis and not receiving $\mathrm{ADT}$ had a lower rate of event-free survival with WPRT (4). A more complete critique of NRG/RTOG 9413 is mentioned below.

In comparison, Sandler et al. analyzed patients with Gleason 9 and 10 diseases only, treated with ADT of various length and either external beam radiotherapy (EBRT) alone, or in combination with brachytherapy (BT). Of the 1,170 patients, $53 \%$ received WPRT. After a reasonably long median follow-up of 5.1 years for EBRT and 6.3 years for BT patients, they found that there was an advantage for WPRT, especially when combined with BT. However, this was not statistically significant as there was a hazard ratio of 0.7 for both biochemical recurrence free survival $(\mathrm{P}=0.07)$ and prostate cancer-specific survival $(\mathrm{P}=0.06)$. The fact that the distant metastasis-free survival, a clinically meaningful endpoint, was not influenced by WPRT (HR 0.9, $\mathrm{P}=0.7$ ), points towards a lack of a clinical benefit for WPRT. Although these results are non-statistically significant, the Kaplan-Meier figure shows that the WPRT plus BT group $(n=318)$ fared the best with a prostate cancer-specific survival rate at 5 years of an astonishing $98 \%$.

The editorial by Chen accompanying the article, titled "Randomized Trials and the Goldilocks Problem", mentioned several important problems (5). However, one has first to define what the goldilocks problem is. For less literary educated people like us who had to google it, the Goldilocks problem comes by analogy to the children's story "Goldilocks and the Three Bears". It refers to a solution that is just right, not too much and not too little and sufficiently "close enough" so that one can stop testing. We believe that the article of Sandler et al. doesn't put WPRT in the "just right" category.

In a similarly interesting article, published within a month of Sandler's article, Tharmalingam et al. (6) studied 812 patients enrolled in a prospective multicenter cohort study in the UK. All patients received a combination of EBRT with high-dose-rate BT and treatment of the pelvis was left to each institution's discretion. In a subset 
analysis, they found that there was an advantage in 5-year progression-free survival in high-risk patients $(84 \%$ vs. $77 \%, \mathrm{P}=0.001$ ), but not in patients with intermediate-risk disease, even when comparing favorable and unfavorable intermediate-risk cancers. In the accompanying editorial, Koontz and Dal Pra (7) state as their main critique of RTOG 9413 that "higher volume disease in the prostate was not entirely eradicated" because of the low 70 Gy dose delivered to the prostate resulting in "reseeding over time" by the primary prostate cancer. Reseeding means that the primary cancer is not sufficiently eradicated and able to "seed" cancer cells to and from distant metastases, a process that has been postulated for some time, especially in breast cancer (8). Therefore, radiotherapy with ADT seems ideal to eliminate oligometastatic subclinical lymph-node disease by a combination of ablative radiation combined with systemic treatments such as ADT (9).

Will patients with positive lymph nodes on PSMAPET be the ones to be treated with WPRT? We believe that PSMA-PET will considerably change our practice of prostate radiotherapy and probably also the way we will address pelvic lymph nodes. In a recently published expedited systematic review comparing PSMA-PET with postoperative pathological results, Petersen et al. (10) found that the positive and negative predictive values of the PSMA-PET were $\geq 80 \%$ in most trials. A radiation oncologist's changes in practice related to PSMA-PET images can be considerable, as many lymph nodes would have been missed if treated without a PSMA PET imaging. Calais et al. (11) found that in 7\% of patients, at least one pelvic lymph node was not covered by the pelvic clinical target volume (CTV). The CTV is defined as the area targeted with radiation, but without the usually added safety margin. In this study, the CTV was contoured without the PSMA-PET information. Thirty-two percent of patients who had N1-disease on standard imaging had at least 1 lesion on PSMA-PET that was not covered by the CTV.

The critique of the two above mentioned randomized trials are well summarized in an editorial by Cozzarini (12): the superior border of the treatment field was too low (S1/S2 and L5/S1, respectively, resulting in insufficient coverage of possibly positive nodes, including patients that had a low risk of metastatic lymph node disease and therefore diluting the statistical result and inadequate dose to microscopic disease. Cozzarini raises an interesting further question of whether WPRT without ADT is less efficient than when combined with ADT. A further interesting point raised by this author was whether WPRT could decrease the immune response by considerably decreasing lymphocystes. As his group has shown in a study that $26 \%$ of patients experiencing grade $\geq 3$ lymphopenia (13) and therefore potentially decreasing the anti-tumor effect of radiation therapy.

Another possible reason for the lack of evidence favoring WPRT in the above mentioned two randomized trials is the possible insufficient radiation dose to the pelvis. GETUG-01 used a dose of 46 Gy in 2 Gy fractions and NRG/RTOG 9413 used a dose of $50.4 \mathrm{~Gy}$ in $1.8 \mathrm{~Gy}$ fractions. If one argues that the radiation dose was insufficient, what about dose-escalation? A phase 2 randomized trial comparing prostate only with WPRT (PIVOTAL trial) is testing this hypothesis of dose escalation (14). Patients in this trial were treated with $60 \mathrm{~Gy}$ in daily fractions of 1.62-Gy to the pelvic lymph nodes.

In radiation oncology, different daily doses are compared with the linear-quadratic (LQ) model to estimate equivalent radiotherapy doses. Different radiation sensitivities of different cells are defined by the $\alpha / \beta$ ratio and incorporated into the LQ model. When assuming an $\alpha / \beta$ ratio of 3 Gy for prostate cancer, this corresponds to an equivalent dose for 2 Gy per fraction of 53.5 Gy compared to calculated 48.4 Gy in NRG/RTOG 9413 and 46 Gy in GETUG-01. The PIVOTAL trial reported more severe patient-reported outcomes and less improvement with time in the WPRT group and more physician assessed grade 2 toxicity with RTOG $\geq$ G2 GI toxicity at 2 years of $24 \%$ with WPRT compared to $16.9 \%$ in the prostate only group.

Koontz and Dal Pra aptly stated in their editorial about WPRT that we await a resolution of the question of WPRT with the publication of RTOG 0924, while in the meantime the discussion is being fed by conflicting data and strong opinions. NRG/RTOG 0924, which is closed to accrual, included patients with unfavorable intermediate and favorable high-risk prostate cancer. All patients randomized to WPRT were treated with a superior border at L4 and a high dose to the prostate. Results are anticipated for 2021. Let's hope that our editorial comment will become obsolete at that time.

\section{Acknowledgments}

Funding: None.

\section{Footnote}

Provenance and Peer review: This article was commissioned by the editorial office, Translational Andrology and Urology. The article did not undergo external peer review. 
Conflicts of Interest: Both authors have completed the ICMJE uniform disclosure form (available at http://dx.doi. org/10.21037/tau-20-881). DT reports grants from Sanofi, outside the submitted work. GD reports grants, personal fees and non-financial support from Janssen Canada, personal fees from Sanofi, personal fees and non-financial support from Bayer, personal fees from Astellas, personal fees from Elekta, personal fees from Ter Sera, personal fees from Abbvie, personal fees from Ferring, other from Progenics, outside the submitted work.

Ethical Statement: The authors are accountable for all aspects of the work in ensuring that questions related to the accuracy or integrity of any part of the work are appropriately investigated and resolved.

Open Access Statement: This is an Open Access article distributed in accordance with the Creative Commons Attribution-NonCommercial-NoDerivs 4.0 International License (CC BY-NC-ND 4.0), which permits the noncommercial replication and distribution of the article with the strict proviso that no changes or edits are made and the original work is properly cited (including links to both the formal publication through the relevant DOI and the license). See: https://creativecommons.org/licenses/by-nc-nd/4.0/.

\section{References}

1. Sandler KA, Cook RR, Ciezki JP, et al. Prostateonly versus whole-pelvis radiation with or without a brachytherapy boost for Gleason grade group 5 prostate cancer: a retrospective analysis. Eur Urol 2020;77:3-10.

2. Avkshtol V, Ruth KJ, Ross EA, et al. Ten-Year Update of a Randomized, Prospective Trial of Conventional Fractionated Versus Moderate Hypofractionated Radiation Therapy for Localized Prostate Cancer. J Clin Oncol 2020;38:1676-84.

3. Roach M, Moughan J, Lawton CAF, et al. Sequence of hormonal therapy and radiotherapy field size in unfavourable, localised prostate cancer (NRG/RTOG 9413): long-term results of a randomised, phase 3 trial. Lancet Oncol 2018;19:1504-15.

4. Pommier P, Chabaud S, Lagrange JL, et al. Is There a Role for Pelvic Irradiation in Localized Prostate Adenocarcinoma? Update of the Long-Term Survival Results of the GETUG-01 Randomized Study. Int J Radiat Oncol Biol Phys 2016;96:759-69.
5. Chen RC. Randomized Trials and the Goldilocks Problem. Eur Urol 2020;77:11-3.

6. Tharmalingam H, Tsang Y, Choudhury A, et al. External Beam Radiation Therapy (EBRT) and High-Dose-Rate (HDR) Brachytherapy for Intermediate and High-Risk Prostate Cancer: The Impact of EBRT Volume. Int J Radiat Oncol Biol Phys 2020;106:525-33.

7. Koontz BF, Dal Pra A. Shifting the Curtain-Can We Make Sense of the Whole Pelvis Controversy? Int J Radiat Oncol Biol Phys 2020;106:534-6.

8. Jagsi R. Progress and controversies: radiation therapy for invasive breast cancer. CA Cancer J Clin 2014;64:135-2.

9. Weichselbaum RR. The 46th David A. Karnofsky Memorial Award Lecture: Oligometastasis-From Conception to Treatment. J Clin Oncol 2018;36:3240-50.

10. Petersen LJ, Zacho HD. PSMA PET for primary lymph node staging of intermediate and high-risk prostate cancer: an expedited systematic review. Cancer Imaging 2020;20:10.

11. Calais J, Kishan AU, Cao M, et al. Potential Impact of 68Ga-PSMA-11 PET/CT on the Planning of Definitive Radiation Therapy for Prostate Cancer. J Nucl Med 2018;59:1714-21.

12. Cozzarini C. Whole-pelvis Radiotherapy in the Radiation Treatment of Intermediate- and High-risk Prostate Cancer: How to Improve the Therapeutic Ratio of a Potentially Effective but still Unsatisfactory Treatment? Eur Urol 2017;71:44-5.

13. Cozzarini C, Noris Chiorda B, Sini C, et al. Hematologic Toxicity in Patients Treated With Postprostatectomy Whole-Pelvis Irradiation With Different Intensity Modulated Radiation Therapy Techniques Is Not Negligible and Is Prolonged: Preliminary Results of a Longitudinal, Observational Study. Int J Radiat Oncol Biol Phys 2016;95:690-5.

14. Dearnaley D, Griffin CL, Lewis R, et al. Toxicity and Patient-Reported Outcomes of a Phase 2 Randomized Trial of Prostate and Pelvic Lymph Node Versus Prostate only Radiotherapy in Advanced Localised Prostate Cancer (PIVOTAL). Int J Radiat Oncol Biol Phys 2019;103:605-17.

Cite this article as: Taussky D, Delouya G. Is pelvic prophylactic radiotherapy in prostate cancer just right? Transl Androl Urol 2020;9(5):2296-2298. doi:10.21037/tau-20-881 